\title{
Editorial for clinical investigations: toward the vision and spirit of CARS with innovative clinical investigations
}

\author{
Heinz U. Lemke ${ }^{1}$
}

Published online: 21 April 2017

(c) CARS 2017

\section{General comments}

At the 20 years celebration of the CARS Congress, which took place in Berlin in 2005, a summary of the impact of CARS-related innovations, and CARS's need for interdisciplinary and international cooperation was presented. The importance of identifying Medical Lead Users, and the validation by these Lead Users of the work achieved by CARS activities, was emphasized.

Medical Lead Users can be defined by the following traits. Typically, Medical Lead Users:

- are aware of the shortcomings of present health care procedures, systems and solutions well before mainstream users,

- are instrumental by themselves to produce innovative (incremental and substantial or transformational) solutions to clinical problems which others have not yet recognized,

- are considered particularly innovative when they find solutions to problems with a high clinical impact,

- are prepared to take risks in an attempt to decrease uncertainty about the outcome of clinical workflows, and

- partner with Lead Innovators and developers.

Since 2005, it has taken another 12 years and the inception of the CARS Journal to start to spotlight these Lead Users and to provide them with a platform to publish their original clinical investigations in a high impact journal with a very interdisciplinary and international readership.

Heinz U. Lemke

hulemke@cars-int.org

1 International Foundation for Computer Assisted Radiology and Surgery (IFCARS), Kuessaberg, Germany
This IJCARS issue includes papers of 11 Lead Users and Clinical Innovators in the fields of Computer Assisted Radiology and Minimally Invasive Therapy (CARMIT), Computer Assisted Orthopaedic Surgery (CAOS) and Computer Assisted Head and Neck Surgery (CANHS). Within the bell distribution of where these fields may be positioned relative to mainstream users (see Fig. 1), they can clearly be identified as early adopters according to E. M. Rogers definition (1983). Note, CARMIT is also an interesting domain in the future for pioneering innovators.

Within each of the given fields for clinical investigations/innovations, there are three aspects which deserve being given special attention in this IJCARS issue:

(1) What novel IT methods, tools and systems are the authors using in their clinical application/feasibility study?

IJCARS has a focus on R\&D results of technical innovations, especially involving novel IT methods, tools and systems, biomedical engineering and medical physics with special emphasis on their applications in radiology and surgery. For novel clinical investigations, this excludes, in principle, commercially available systems or generally available open source software systems (i.e., applying a set of standard algorithms and tools), unless they are employed in a very innovative clinical context. Connected to this aspect is also a question of special interest to the CARS community, i.e., what type of R\&D for improving the methods/tools and their clinical applications have the authors envisaged for the future?

(2) Impact on diagnostic/therapeutic Workflow, i.e., what is the effective innovation within clinical procedures?

How are the clinical workflows affected by the investigated technologies? For example, which activities in 


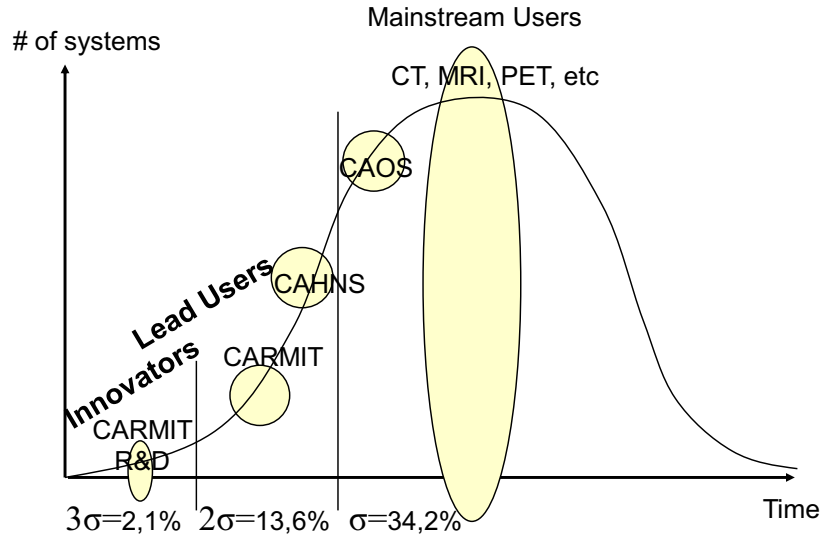

Fig. 1 Lead Users and Innovators of CARS as early adopters versus mainstream users

a given workflow become IT based and what are the clinical and organizational changes implied with the successful introduction of these procedures in a clinical setting? What will be the potential degree of workflow automation possible using workflow management systems?

Managing changes to a traditional clinical workflow are a difficult hurdle to undertake, particularly, if they are in conflict with the gold standard Evidence-Based Medicine (EBM). For example, who would be prepared to provide Model-Based Medical Evidence (MBME) for patient-specific decision making a higher priority than classic, i.e., clinical trial-based (but non-patientspecific) decision making?

(3) How do these methods/tools potentially impact clinical outcome for the patient, i.e., what is the expected/verified innovation for improved outcomes and their statistical significance?

Typical examples for this aspect relate to features such as procedural invasiveness, duration and accuracy, reducing the effective radiation dose for the patient (and physician), volume of contrast agent, better metrics on resection margins during surgery, reduction in intraoperative blood loss/hemorrhage or reduction in iatrogenic damage and preservation of critical structures.

Ideally, in order to be publishable, all of these features need to be supported by reproducible statistics and/or the implementation of some de-biasing strategies. Well-designed and well-conducted clinical investigation should eliminate or minimize clinical biases (which potentially can be a long list). For example, groups of patients for treatment and control need to be randomized in order to increase the validity of a study. If a clinical study relies on patient self-assessment or physician assessment of patient status, it is susceptible to assessment bias.
A publication should show awareness of the relevant biases and point out which de-biasing steps have been taken. To ascertain statistical significance by some mathematical means is certainly a valuable approach but in case of "small numbers", which frequently can be observed for innovative clinical investigations, this may not always be realizable and should therefore be appropriately considered in clinical judgment and decision making.

The following contains a brief synopsis of eleven papers in the field of CARMIT, CAOS and CANHS published in this IJCARS issue, in the light of the three aspects indicated above.

\section{Computer Assisted Radiology and Minimally Invasive Therapy (CARMIT)}

(1) Evaluation of a robotic system for irreversible electroporation (IRE) of malignant liver tumors: initial results

L. P. Beyer ${ }^{1}$, B. Pregler ${ }^{1}$, K. Michalik ${ }^{1}$, C. Niessen ${ }^{1}$, M. Dollinger $^{1}$, M. Müller ${ }^{2}$, H. J. Schlitt ${ }^{3}$, C. Stroszczynski ${ }^{1}$, P. Wiggermann ${ }^{1}$

${ }^{1}$ Department of Radiology

${ }^{2}$ Department of Internal Medicine I

${ }^{3}$ Department of Surgery, University Medical Center Regensburg, Germany

A novel robotic system for IRE of the liver is presented, comparing the manual with the robot-supported workflow. Important outcome criteria selected relate to the procedural duration and accuracy but also to the reduction in radiation dose for the physician and the patient. The validity of this study is based on 35 patients (40 cases) and is investigated with the JMP statistics software package. Considering that the study comes from a single-center set-up with a low number of procedures (19 manual and 21 robotic), it limits generalization of the given very promising results.

(2) Computer-aided evaluation of low-dose and low-contrast agent third-generation dual-source $\mathrm{CT}$ angiography prior to transcatheter aortic valve implantation (TAVI)

Peter Dankerl ${ }^{1}$, Matthias Hammon ${ }^{1}$, Hannes Seuss ${ }^{1}$, Monique Tröbs ${ }^{2}$, Annika Schuhbaeck ${ }^{2}$, Michaela M. Hell $^{2}$, Alexander Cavallaro ${ }^{1}$, Stephan Achenbach ${ }^{2}$, Michael Uder ${ }^{1}$, Mohamed Marwan ${ }^{2}$

${ }^{1}$ Department of Radiology

${ }^{2}$ Department of Cardiology, Universitätsklinikum Erlangen, Friedrich-Alexander-Universität Erlangen-Nürnberg, Germany 
A commercially available software package for computeraided evaluation of angiograms from a third-generation dual-source CT system is being employed to produce a variety of metrics that can impact the TAVI workflow. Important outcome criteria indicate a systematic overestimation of the computer-aided evaluation for one specific metric, but this has been achieved with half the volume of contrast agent and less than one-fifth of the effective radiation dose. The validity of this study is based on 30 patients and is investigated with the SPSS statistics software package.

(3) Quantification of motion of the thoracic aorta after ascending aortic repair of type-A dissection

Ga-Young Suh ${ }^{1}$, Dominik Fleischmann², Ramin E. Beygui $^{3}$, Christopher P. Cheng ${ }^{1}$

${ }^{1}$ Department of Surgery

${ }^{2}$ Department of Radiology

${ }^{3}$ Department of Cardiothoracic Surgery, Stanford University, USA

A custom software package for creating patient-specific 3D models and geometric measurements from CTAs to better understand the workflow implied in graft repair of ascending aortic dissections. It is concluded that the relationship between geometric measurements and clinical outcomes is not well understood. The validity of this study, which is based on only eight patients with no statistical analysis, is very limited.

(4) Evaluation of tongue squamous cell carcinoma resection margins using ex vivo MR

Stefan C. A. Steens ${ }^{1}$, Elise M. Bekers ${ }^{2}$, Willem L. J. Weijs $^{3}$, Geert J. S. Litjens ${ }^{2}$, Andor Veltien ${ }^{1}$, Arie Maat ${ }^{2}$, Guido B. van den Broek ${ }^{4}$, Jeroen A. W. M. van der Laak $^{2}$, Jürgen J. Fütterer ${ }^{1}$, Christina A. Hulsbergen van der $\mathrm{Kaa}^{2}$, Matthias A.W. Merkx ${ }^{3}$, Robert P. Takes ${ }^{4}$

${ }^{1}$ Department of Radiology and Nuclear Medicine

${ }^{2}$ Department of Pathology

${ }^{3}$ Department of Oral and Maxillofacial Surgery

${ }^{4}$ Department of Otorhinolaryngology and Head and Neck Surgery, Radboud, University Medical Center, Nijmegen, The Netherlands

Digital photography and ex vivo 7T MR are being used to facilitate matching between histopathology slices (as the gold standard) and MR images. Registration is done by hand, but it is envisaged that for the surgical workflow of TSCC resection with a 3T MR located in the operating room and appropriate rigid and/or non-rigid registering of MR and histopathology data by post-processing software, a much better outcome for patients as regards minimal resection margin may be possible. To achieve this, the authors seek collaborations with industrial partners and technical and IT experts. The validity of this study, which is based on only ten patients with no statistical analysis, is very limited.

\section{Computer Assisted Orthopaedic Surgery (CAOS)}

(1) Comparison of rotational acetabular osteotomy performed with navigation by surgeons with different levels of experience of osteotomies

Masaki Takao ${ }^{1}$, Takashi Nishii ${ }^{1}$, Takashi Sakai ${ }^{2}$, Nobuhiko Sugano ${ }^{1}$

${ }^{1}$ Department of Orthopaedic Medical Engineering

${ }^{2}$ Department of Orthopaedic Surgery, Osaka University, Japan

A custom polygon viewer program using an open source software system was developed and is assessed for surgical navigation in rotational acetabular osteotomy by surgeons with different levels of experience. The outcome showed no significant differences between the high-experience and low-experience surgeons in regard to the incision length, operation time or intraoperative blood loss. The validity of this study is based on 24 patients and is statistically investigated for the two groups of surgeons with the Mann-Whitney $U$-test and Fisher's exact probability test for comparing continuous and nominal variables, respectively.

(2) Central X-ray beam correction of radiographic acetabular cup measurement after THA: an experimental study

T. Schwarz ${ }^{1}$, M.Weber $^{1}$, M. Wörner ${ }^{1}$, T. Renkawitz ${ }^{1}$, J. Grifka $^{1}$, B. Craiovan ${ }^{1}$

${ }^{1}$ Department of Orthopedic Surgery, Regensburg University, Germany

The paper describes an experimental fluoroscopic set-up with an artificial acetabular cup holder in order to compare the methods developed by Lewinnek and Widmer to measure and differentiate retroversion from anteversion taking into account the central X-ray beam offset. Highly accurate measurement of the cup position is critical for evaluating outcome after total hip arthroplasty (THA), in particular for the assessment of cup orientation on postoperative radiographs. The validity of this study is based on 24 radiographs manually analyzed by three examiners and statistically investigated by means of the Mann-Whitney test for nonparametric testing. It is hoped that future computer-assisted radiology CAD software will provide immediate central beam correction during the measurement of parameters. 
(3) Analysis of acetabular orientation and femoral anteversion using images of three-dimensional reconstructed bone models

Jaeyeong Park ${ }^{1}$, Jun-Young Kim ${ }^{2}$, Hyun Deok Kim ${ }^{3}$, Young Cheol $\mathrm{Kim}^{3}$, Anna $\mathrm{Seo}^{3}$, Minkyu Je${ }^{4}$, Jong Uk Mun ${ }^{5}$, Bia Kim, Il Hyung Park ${ }^{2}$, Shin-Yoon Kim ${ }^{2}$

1 Division of Biomedical Science, Kyungpook National University, Daegu

${ }^{2}$ Department of Orthopedic Surgery, Kyungpook National University Hospital

${ }^{3}$ Institute of Advanced Convergence Technology, Kyungpook National University, Daegu

${ }^{4}$ School of Electrical Engineering, Korea Advanced Institute of Science and Technology, Daejeon

${ }^{5}$ Department of Orthopedic Surgery, Changwon Gyeongsang, National University, Republic of Korea

To evaluate outcome after THA based on anthropometric information, this paper focuses on 3D reconstructed models for acetabular inclination and anteversion as well as for femoral anteversion. The validity of this study is based on CT data from 96 patients. Two orthopedic surgeons participated in measuring and evaluating the differences between 2D slice images and 3D measurements. It could be shown that intra- and inter-rater reliabilities for 3D measurements were more consistent than those for measurements using 2D slice images. The statistical analysis was performed using IBM Statistics 22.0

(4) Application of computer-aided design and 3D-printed navigation template in Locking Compression Pediatric Hip PlateTM placement for pediatric hip disease

Pengfei Zheng ${ }^{1,2}$, Qingqiang $\mathrm{Yao}^{2,3}$, Peng Xu ${ }^{1,2}$, Liming Wang $^{2,3}$

${ }^{1}$ Department of Pediatric Orthopaedics, Children's Hospital, Nanjing Medical University

${ }^{2}$ Digital Medicine Institute, Nanjing Medical University

${ }^{3}$ Department of Orthopedics, Nanjing First Hospital, Nanjing Medical University, China

There are few examples which can demonstrate that preoperative $3 \mathrm{D}$ modeling and computer simulation combined with computer-aided design (CAD) and 3D printing technology can improve more effectively the efficiency and safety in orthopedic surgery, than the example in pediatric surgery provided in this paper. These methods and tools appear to be essential for the accurate placement of screws in Locking Compression Pediatric Hip Plate (LCP-PHP) surgery. Even though only 11 patients served as the sample base for this intervention, compared with a conventional surgery group $(n=13)$, there was a statistically significant reduction in intraoperative damage and intraoperative hemorrhage, decrease in operation time as well as a decrease in radiation exposure to patients and personnel during the surgery, $p$ value $<0.05$.

(5) Feasibility of laser-guided percutaneous pedicle screw placement in the lumbar spine using a hybrid-OR

P. H. Richter ${ }^{1}$, F. Gebhard ${ }^{1}$, M. Salameh ${ }^{1}$, K. Schuetze ${ }^{1}$, M. Kraus ${ }^{2}$

${ }^{1}$ Department of Trauma-, Hand- and Reconstructive Surgery, Ulm University

${ }^{2}$ Centre for Orthopaedic, Trauma and Spine Surgery, Donau-Ries Hospital Donauwoerth, Germany

Intraoperative imaging combined with robot-assisted pedicle screw placement can be cost saving due to a distinct reduction in revision surgery, but procedure time for the new technique, as shown in this paper, can be significantly longer in comparison with the standard technique. It can, however, be also of an additional help for screw implantation in challenging regions with poor visualization. The study presented in the paper was carried out on ten lumbar spine models of which five were positioned on an operating table in a hybrid-OR with a new laserguided technique used to insert the pedicle screws, the other five models were used for standard fluoroscopic screw implantation. GraphPad PRISM ${ }^{\circledR} 5$ was used for the statistical analysis with the Mann-Whitney- $U$ test to assess differences in the guided group versus the nonguided group.

\section{Computer Assisted Head and Neck Surgery (CANHS)}

(1) Intraoperative navigation in complex head and neck resections: indications and limits

S. Catanzaro ${ }^{1}$, C. Copelli $^{1}$, A. Manfuso ${ }^{2}$, K. Tewfik $^{1}$, N. Pederneschi ${ }^{1}$, L. Cassano ${ }^{1}$, R. Cocchi ${ }^{1}$

${ }^{1}$ Operative Unit of Maxillo-Facial Surgery, Otolaryngology and Dentistry, Head and Neck Department, Hospital Casa Sollievo della Sofferenza

${ }^{2}$ Operative Unit of Maxillo-Facial Surgery, Federico II University, Naples, Italy

This paper describes an intraoperative CT-based navigation for the resection of head and neck tumors to overcome the challenging task of handling the anatomical complexity and the functional importance of the structures involved. A sample of 16 patients was divided in a "study group" treated with CT computer-assisted surgery and a "control group" surgically treated without the use of this technology. The "study group" showed a 
reduced rate of errors in the histological specimen orientation and an increased distance of the tumor from the margins of resection, thereby improving the oncological safety for a selected group of patients. The objective of this prospective pilot study (with no statistical analysis) was to develop a protocol to be validated on a larger group of patients in follow-up studies.

(2) Minimally invasive, multi-port approach to the lateral skull base: a first in vitro evaluation

Igor Stenin ${ }^{1}$, Stefan Hansen ${ }^{2}$, M. Nau-Hermes ${ }^{3}$, W. El-Hakimi ${ }^{4}$, M. Becker ${ }^{4}$, J. Bredemann ${ }^{3}$, J. Kristin ${ }^{1}$, T. Klenzner ${ }^{1}$, J. Schipper ${ }^{1}$

${ }^{1}$ Department of Otorhinolaryngology, University Hospital Düsseldorf

${ }^{2}$ Department of Otorhinolaryngology, University Hospital Essen

${ }^{3}$ Laboratory for Machine Tools and Production Engineering, RWTH Aachen University
${ }^{4}$ Interactive Graphics Systems Group, Technical University Darmstadt, Germany

The presented workflow and tools of an experimental self-developed positioning system allow for the planning and drilling of multiple minimally invasive canals at the lateral skull base. This preclinical study was carried out on one dummy model and one cadaver skull, with three drilling trajectories. Although there is no statistical analysis presented, it is postulated that the given results potentially allow for a reduction in iatrogenic damage and preservation of critical structures. Before exhaustive clinical studies are performed, further optimization of the drilling process for reducing the drilling error of the system may have to take place.

\section{Compliance with ethical standards}

Conflict of interest The author declares that he has no competing interests. 